\title{
A Meta-Analysis for Using Radiomics to Predict Complete Pathological Response in Esophageal Cancer Patients Receiving Neoadjuvant Chemoradiation
}

\author{
YUNG-SHUO KAO ${ }^{1}$ and YEN HSU ${ }^{2}$ \\ ${ }^{1}$ Department of Radiation Oncology, China Medical University Hospital, Taichung, Taiwan, R.O.C.; \\ ${ }^{2}$ Department of Family Medicine, Changhua Christian Hospital, Changhua, Taiwan, R.O.C.
}

\begin{abstract}
Background: Preservation of organ function is important in cancer treatment. The 'watch-and-wait' strategy is an important approach in management of esophageal cancer. However, clinical imaging cannot accurately evaluate the presence or absence of residual tumor after neoadjuvant chemoradiation. As a result, using radiomics to predict complete pathological response in esophageal cancer has gained in popularity in recent years. Given that the characteristics of patients and sites vary considerably, a meta-analysis is needed to investigate the predictive power of radiomics in esophageal cancer. Patients and Methods: PRISMA guidelines were used to conduct this study. PubMed, Cochrane, and Embase were searched for literature review. The quality of the selected studies was evaluated by the radiomics quality score. $I^{2}$ score and Cochran's $Q$ test were used to evaluate heterogeneity between studies. A funnel plot was used for evaluation of publication bias. Results: A total of seven articles were collected for this meta-analysis. The pooled area under the receiver operating characteristics curve of the seven selected articles for predicting pathological complete response in eosphageal cancer patient was quite high, achieving a pooled value of 0.813 (95\% confidence intervaI $=0.761-0.866)$. The radiomics quality score ranged from -2 to 16 (maximum score: 36 points). Three out of the seven studies used machine learning algorithms, while the others used traditional biostatistics methods. One of the seven studies used morphology class features, while four studies used first-order features, and five used second-order
\end{abstract}

This article is freely accessible online.

Correspondence to: Yung-Shuo Kao, No. 2, Yude Rd., North Dist., Taichung City 404332, Taiwan, R.O.C. E-mail: codingforlifetime@gmail.com

Key Words: Esophageal cancer, meta-analysis, radiomics. features. Conclusion: Using radiomics to predict complete pathological response after neoadjuvant chemoradiotherapy in esophageal cancer is feasible. In the future, prospective, multicenter studies should be carried out for predicting pathological complete response in patients with esophageal cancer.

Esophageal cancer is a common gastrointestinal malignancy that causes more than 500,000 cancer deaths per year (1). The 5-year survival rate of patients with esophageal cancer is less than $25 \%$ (1). Developing a better treatment strategy for esophageal cancer is important.

The current treatment strategy for locally advanced esophageal cancer is neoadjuvant chemoradiotherapy followed by surgery. If complete metabolic response follows neoadjuvant treatment, then an active surveillance strategy can be considered (2). However, with the commonly used metabolic imaging methods, such as positron-emitted tomography/computed tomography, it is difficult to differentiate between inflammation and residual malignancy after concurrent chemoradiotherapy. In addition, residual malignancy is not always detected by PET/CT scan $(3,4)$. Other imaging methods, such as magnetic resonance imaging scan, and computed tomography alone, also have the same problem $(3,4)$. For this reason, attention has turned to using radiomics to predict complete pathological response in order to help patients and physicians to choose the best treatment approach when the watch-and-wait strategy is being considered.

Radiomics is a quickly growing field in which clinical images are transformed into quantitative features. These radiomics features can be further used to predict clinical outcomes. In esophageal cancer, radiomics has been widely used, as a previous review has reported (5). However, radiomics features were found to be associated with the imaging equipment used, technical setting, and processing kernel (6). As a result, there is an unmet need to conduct a meta-analysis to investigate the pooled predictive power of 


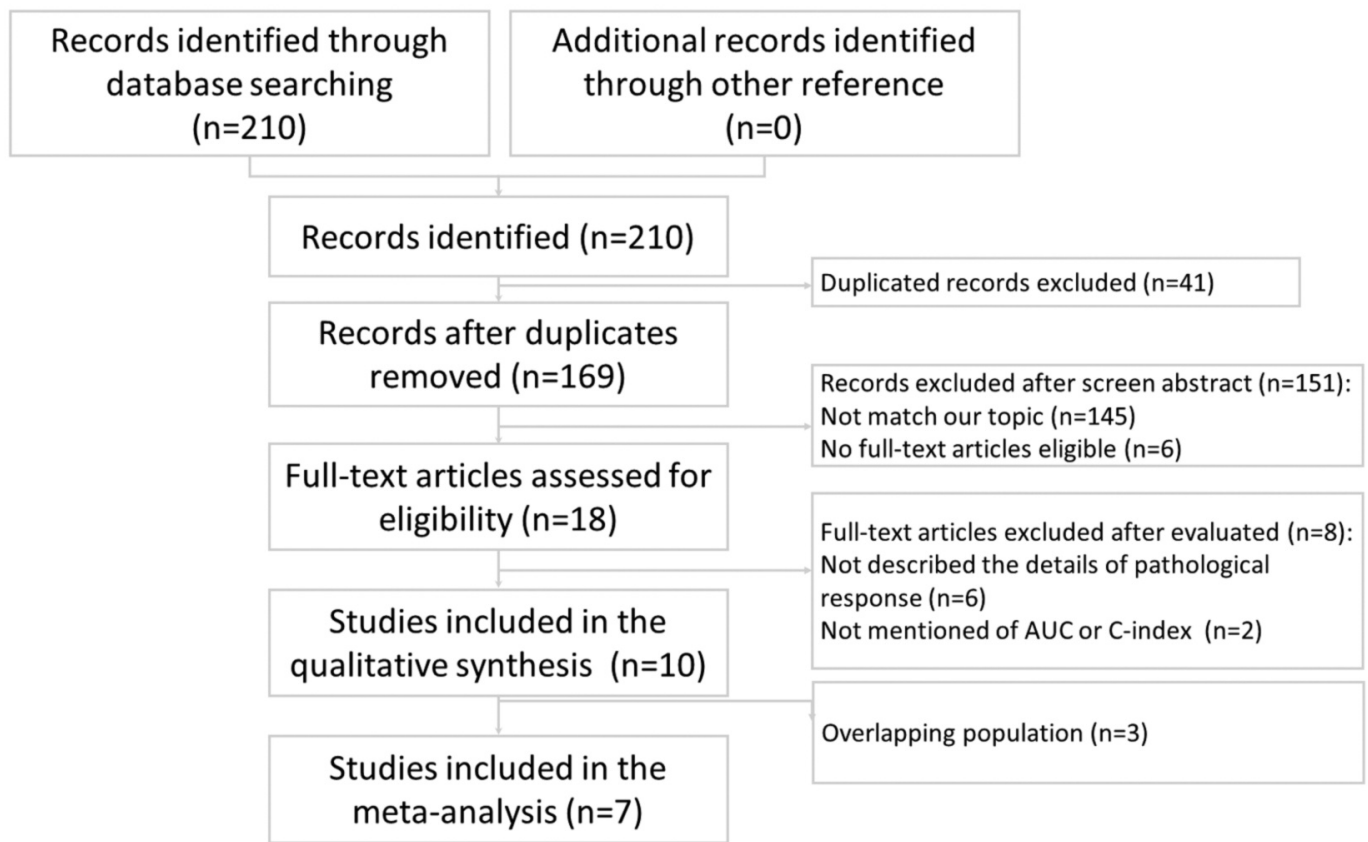

Figure 1. The article collection flowchart.

the current model. The results can be used as a benchmark for future large-scale radiomics studies for predicting complete pathological response in esophageal cancer.

\section{Patients and Methods}

PRISMA guidelines were used to conduct this study (7).

Eligibility criteria. Articles were included according to the rules provided below: i) Patients with esophageal cancer received neoadjuvant chemoradiation. ii) Radiomics was used to predict complete pathological response versus other responses and to report the area under the receiver operating characteristics curve (AUC). The radiomics features were defined in (8) as the Image Biomarker Standardization Initiative. iii) Full text article. iv) Article in English. v) Published up to 2021 .

We excluded articles according to the rules provided below: i) Abstract only or short communication (9). ii) Deep learning only was used to generate features.

Literature search. The Authors searched PubMed, Cochrane, and Embase using key words: (esophageal cancer) AND (radiomics OR texture OR histogram) AND (response OR remission).

Data extraction. We extracted the primary endpoint, which was set as the highest AUC in the validation set (testing set). When there was no external validation set, the result from the internal validation set was chosen. When there was no internal validation, the result from the training set (development set) was chosen. When the result reported the C-index, it was used as the AUC. The collected model had to contain radiomics-related features but was also allowed to contain other features, such as clinical or pathological features.

We also extracted other information, including the region of interest, patient number, image type, radiomics feature type, and algorithm used in the outcome prediction.

Statistical analysis. The random-effects model was used to calculate the pooled AUC and to form the forest plot. The calculation of pooled AUC requires the standard error of the AUC; therefore, when the value was not reported in the literature, we used the formula proposed by Hanley and McNeil to calculate it (10). We also evaluated the study heterogeneity by $I^{2}$ score and Cochran's Q test. $I^{2}$ scores of less than $50 \%$ mean that there was low to moderate heterogeneity between the studies; otherwise, there is high heterogeneity between them. $p$-Values of less than 0.05 indicate statistical significance. The confidence interval calculation of the AUC was calculated using Excel (Microsoft, Redmond, Washington, MA, USA); all other statistics were accomplished using MedCalc (version 19.6.1; MedCalc, Acacialaan, Ostend, Belgium).

Risk of bias. Bias between the selected studies in meta-analysis was measured using a funnel plot. Since the number of collected articles was smaller than 10, we were unable to use Egger's test according to the recommendation provided by the Cochrane handbook (11).

Quality assessment. Study quality was evaluated by the radiomics quality score (RQS) (12). The quality of the selected literature was evaluated by both Authors. The inter-rater intra-class correlation was calculated based on the total RQS in order to evaluate interrater reliability (13). 
Table I. The details of articles in the quantitative meta-analysis.

\begin{tabular}{|c|c|c|c|c|c|c|c|}
\hline Author (Ref) & Country, year & Cancer & ROI & Imaging & $\begin{array}{c}\text { Training } \\
\text { set }\end{array}$ & $\begin{array}{c}\text { External } \\
\text { validation }\end{array}$ & $\begin{array}{c}\text { Highest } \\
\mathrm{AUC} \pm \mathrm{SE}\end{array}$ \\
\hline Beukinga et al. (14) & NL, 2017 & $\mathrm{EC}$ & GTV & PET & 97 & No & $0.74 \pm 0.05$ \\
\hline Beukinga et al. (15)* & NL, 2018 & $\mathrm{EC}$ & GTV & PET & 73 & No & $0.81 \pm 0.05$ \\
\hline Beukinga et al. (16)* & NL, 2020 & $\mathrm{EC}$ & GTV & PET & 96 & No & $0.70 \pm 0.06^{\mathrm{a}}$ \\
\hline Hu et al. (17) & CHN, 2020 & $\mathrm{EC}$ & $\begin{array}{c}\text { GTV } \\
\text { periGTV }\end{array}$ & $\mathrm{CT}$ & 161 & 70 & $0.88 \pm 0.05$ \\
\hline Hu et al. (18)* & CHN, 2020 & $\mathrm{EC}$ & GTV & $\mathrm{CT}$ & 161 & 70 & $0.725 \pm 0.01$ \\
\hline Rossum et al. (19) & USA, 2016 & $\begin{array}{c}\text { EC } \\
\text { EGJC }\end{array}$ & GTV & PET & 217 & No & $0.77 \pm 0.03^{b}$ \\
\hline Hirata et al. (20) & JPN, 2020 & $\mathrm{EC}$ & GTV & MRI & 58 & No & $0.86 \pm 0.08$ \\
\hline Yang et al. (21) & CHN, 2019 & $\mathrm{EC}$ & GTV & $\mathrm{CT}$ & 44 & 11 & $0.79 \pm 0.16$ \\
\hline Rishi et al. (22) & USA, 2020 & $\begin{array}{c}\text { EC } \\
\text { EGJC }\end{array}$ & GTV & $\begin{array}{l}\text { PET } \\
\text { CT }\end{array}$ & 68 & No & $0.87 \pm 0.01$ \\
\hline Yip et al. (23) & USA, 2016 & $\mathrm{EC}$ & GTV & PET & $45(30)$ & No & $0.65 \pm 0.10^{c}$ \\
\hline
\end{tabular}

AUC: Area under the receiver operating characteristics curve; CHN: China; CT: computed tomography; EC: esophageal cancer; EGJC: esophagealgastric junction cancer; GTV: gross tumor volume; JPN: Japan; MRI: magnetic resonance imaging; NL: Netherlands; periGTV: peritumoral area;

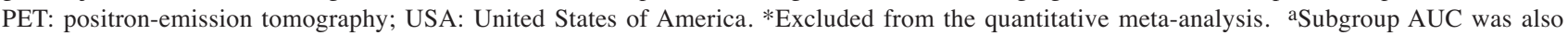
reported but AUC for the whole group, which was higher, was used here. ${ }^{b} \mathrm{C}$-index was reported. cTotal of 45 patients; area under the curve is reported here for eight with complete response versus 22 partial response.

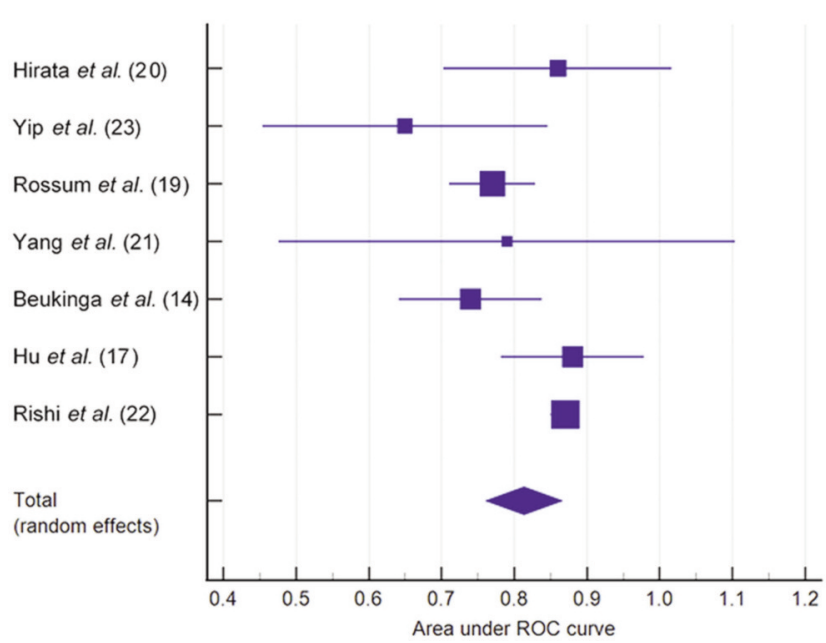

Figure 2. Forest plot for the area under the receiver operating characteristics (ROC) curve for predicting pathological complete response in patients with esophageal cancer.

\section{Results}

A total of 210 articles were gathered. The article selection flowchart is provided in Figure 1. After the selection process, 10 articles remained for qualitative meta-analysis. Three articles were conducted by Beukinga et al. (14-16), therefore we only chose one of these for further analysis. Two articles were conducted by $\mathrm{Hu}$ et al. $(17,18)$, therefore we selected only one of them for the subsequent analysis. As a result,

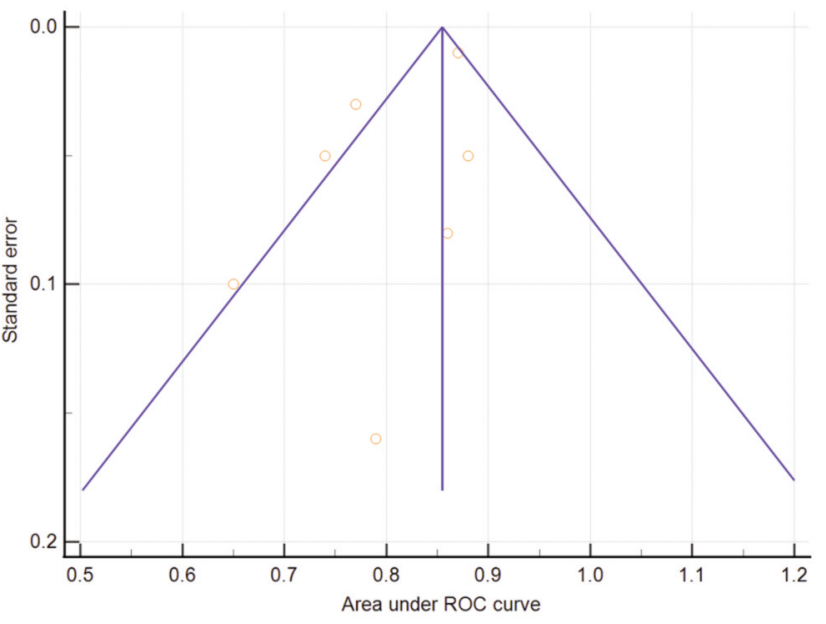

Figure 3. Funnel plot.

seven articles were chosen for quantitative meta-analysis. The details of the collected literature are listed in Table I. All articles extracted radiomics features from the gross tumor volume. In addition, one article also extracted radiomics features from the peri-tumoral area.

Overall literature assessment. The pooled AUC for the seven collected studies was 0.813 (95\% confidence intervaI $=0.761$ $0.866)$. The forest plot is provided in Figure 2. The $I^{2}$ score 
Table II. Radiomics Quality Score table.

\begin{tabular}{|c|c|c|c|c|c|c|c|}
\hline Criteria/study & $\begin{array}{l}\text { Beukinga } \text { et al. } \\
\text { (14) }\end{array}$ & $\begin{array}{l}\text { Hu et al. } \\
\quad \text { (17) }\end{array}$ & $\begin{array}{l}\text { Rossum et al. } \\
\text { (19) }\end{array}$ & $\begin{array}{l}\text { Hirata et al. } \\
\qquad(20)\end{array}$ & $\begin{array}{l}\text { Yang et al. } \\
\quad(21)\end{array}$ & $\begin{array}{l}\text { Rishi et al. } \\
\quad \text { (22) }\end{array}$ & $\begin{array}{l}\text { Yip et al. } \\
\quad \text { (23) }\end{array}$ \\
\hline Image protocol quality & 2 & 1 & 1 & 1 & 1 & 1 & 1 \\
\hline Multiple segmentations & 1 & 1 & 1 & 0 & 0 & 2 & 0 \\
\hline Phantom study on all scanners & 0 & 0 & 0 & 0 & 0 & 0 & 0 \\
\hline Imaging at multiple time points & 0 & 0 & 0 & 1 & 1 & 0 & 0 \\
\hline $\begin{array}{l}\text { Feature reduction or adjustment } \\
\text { for multiple testing }\end{array}$ & 3 & 3 & 3 & -3 & 3 & 3 & 3 \\
\hline $\begin{array}{l}\text { Multivariable analysis with } \\
\text { non-radiomics features }\end{array}$ & 1 & 1 & 1 & 0 & 0 & 0 & 0 \\
\hline Detect and discuss biological correlates & 0 & 1 & 0 & 0 & 0 & 0 & 0 \\
\hline Cut-off analyses & 0 & 1 & 0 & 1 & 0 & 0 & 1 \\
\hline Discrimination statistics & 2 & 1 & 2 & 1 & 2 & 2 & 1 \\
\hline Calibration statistics & 2 & 1 & 2 & 0 & 0 & 0 & 0 \\
\hline $\begin{array}{l}\text { Prospective study registered } \\
\text { in a trial database }\end{array}$ & 0 & 0 & 0 & 0 & 0 & 0 & 0 \\
\hline Validation & 2 & 3 & 2 & -5 & 2 & 2 & -5 \\
\hline Comparison to 'gold standard' & 0 & 0 & 2 & 0 & 0 & 0 & 2 \\
\hline Potential clinical utility & 2 & 2 & 2 & 2 & 2 & 2 & 2 \\
\hline Cost-effectiveness analysis & 0 & 0 & 0 & 0 & 0 & 0 & 0 \\
\hline Open science and data & 0 & 0 & 0 & 0 & 0 & 0 & 0 \\
\hline $\begin{array}{l}\text { Total score (Maximum score: } \\
36 \text { points) }\end{array}$ & 15 & 15 & 16 & -2 & 11 & 12 & 5 \\
\hline
\end{tabular}

was $70.28 \%$ (Cochran's Q test: $p=0.0026$ ), which means that high heterogeneity existed within our studies. The funnel plot appeared asymmetric (Figure 3). However, because we only included seven studies in our meta-analysis, we cannot determine whether publication bias exists or not by examining the funnel plot.

Quality of radiomics studies. The quality of each of the seven studies was assessed by RQS, as seen in Table II. The intraclass correlation between the two reviewers who independently evaluated the articles was $0.9317(95 \%$ confidence interval $=0.6027-0.9883$ ). High intra-class correlation means that our quality assessment was reliable. Inconsistencies were discussed by the reviewers, and the finalized results (Table II) were reached by consensus. The RQS ranged from -2 to 16 in the selected studies. Given that the maximum was 36 points, the highest-rated study received only $40 \%$ of the points.

Review of type of radiomics feature and other features in selected studies. According to International Symposium on Biomedical Imaging (ISBI) standards, the radiomics features can be divided into morphology class, first-order class, and second-order class. The second-order class includes the graylevel (GL) co-occurrence matrix, GL run-length matrix, GL size-zone matrix, GL distance-zone matrix, neighborhood gray tone difference matrix, and neighboring GL dependence
Table III. Features used in the model with the highest area under the curve of the selected studies.

\begin{tabular}{|c|c|c|}
\hline Author (Ref) & Radiomics feature type & Other features \\
\hline $\begin{array}{l}\text { Beukinga } \\
\text { et al. (14) }\end{array}$ & GLRLM & $\begin{array}{l}\text { Histology, } \\
\text { clinical T stage }\end{array}$ \\
\hline $\begin{array}{l}\mathrm{Hu} \\
\text { et al. (17) }\end{array}$ & $\begin{array}{l}\text { First-order class, } \\
\text { GLCM, GLSZM }\end{array}$ & None \\
\hline $\begin{array}{l}\text { Rossum } \\
\text { et al. (19) }\end{array}$ & $\begin{array}{l}\text { Morphology class, } \\
\text { first-order class }\end{array}$ & None \\
\hline $\begin{array}{l}\text { Hirata } \\
\text { et al. }(20)\end{array}$ & First-order class & None \\
\hline $\begin{array}{l}\text { Yang } \\
\text { et al. (21) }\end{array}$ & First-order class, GLRLM & None \\
\hline $\begin{array}{l}\text { Rishi } \\
\text { et al. (22) }\end{array}$ & $\begin{array}{c}\text { First-order class, GLRLM, GLSZM, } \\
\text { GLDZM, fractal feature* }\end{array}$ & None \\
\hline Yip et al. (23) & GLCM, GLRLM, GLSZM & None \\
\hline
\end{tabular}

*Fractal feature is not listed as a type of International Symposium on Biomedical Imaging feature (8). GLRLM: Gray-level run-length matrix; GLCM: Gray-level co-occurrence matrix; GLSZM: Gray-level sizezone matrix; GLDZM: Gray-level distance-zone matrix.

matrix (8). We reviewed the radiomics feature type used and other types of features of the selected articles, and the results are provided in Table III. The feature type provided in the table is the feature used in the model with the highest AUC. One of the seven studies used the morphology class feature 
Table IV. Algorithms used in the predictive model.

\begin{tabular}{lc}
\hline Author (Ref) & Algorithm \\
\hline $\begin{array}{l}\text { Beukinga } \text { et al. (14) } \\
\text { Hu } \text { et al. (17) }\end{array}$ & $\begin{array}{c}\text { Multivariate logistic regression with LASSO } \\
\text { Support vector machine with radial basis kernel } \\
\text { Mossum } \text { et al. (19) }\end{array}$ \\
$\begin{array}{l}\text { Multivariable logistic regression with } \\
\text { stepwise backward elimination }\end{array}$ \\
$\begin{array}{l}\text { Yang } \text { et al. } \text { (20) }(21) \\
\text { Rishi } \text { et al. } \text { (22) }\end{array}$ & Multivariate logistic regression with LASSO \\
Yip et al. (23) & Kaplan-Meier method with log-rank test \\
\hline
\end{tabular}

LASSO: Least absolute shrinkage and selection operator.

(19), four studies used the first-order feature $(17,20-22)$, and five studies used the second-order feature $(14,17,21-23)$.

Review of the algorithms used in predictive models. The details of the algorithms used in the selected studies are provided in Table IV. Of the selected studies, three used machine learning algorithms (LASSO and SVM) $(14,17,21)$, while the others used traditional biostatistical methods $(19,20,22,23)$.

\section{Discussion}

To our knowledge, this study is the first meta-analysis of the use of radiomics to predict a complete pathological response after neoadjuvant chemoradiotherapy in esophageal cancer. The pooled AUC of the seven selected articles was found to be quite high, at $0.813(95 \% \mathrm{CI}=0.761-0.866)$, and the $I^{2}$ score was $70.28 \%(p=0.0026)$. High heterogeneity existed between the studies, which is reasonable given that radiomics features are influenced by imaging equipment, technical setting, and processing kernel (6). The funnel plot shows a symmetric pattern, indicating no publication bias between the selected studies.

Although the predictive power of radiomics was good, the overall quality of the selected studies was poor. The radiomics quality score ranged from -2 to 16 (maximum score: 36 points). Although RQS is widely used in evaluation of the quality of radiomics studies, some of its items are difficult to achieve. For example, it is difficult to conduct analysis of cost-effectiveness for radiomics in cancer. In a radiomics review about lung cancer, among the 14 collected studies, none had performed a cost-effectiveness analysis (24). In addition, carrying out phantom studies for all scanners for radiomics studies is not routine. In a review of renal cell carcinoma studies, none of the 57 studies had performed phantom studies for use of different scanners (25). None of the studies included in this meta-analysis of esophageal cancer performed a cost-effectiveness analysis or carried out phantom studies.
Five studies in our analysis used second-order radiomics features in the predictive model. Second-order radiomics features show the inter-voxel relationship, which can be used to evaluate intra-tumoral homogeneity (26). The intratumoral heterogeneity was shown to be correlated to tumor resistance in many cancer types, such as glioblastoma (27) and lung cancer (28).

A machine learning algorithm was used in three out of the seven studies. The machine learning approach has become highly popular in recent years. However, none of the selected studies used deep-learning techniques. This is not surprising as deep learning requires a large sample size to demonstrate its power (29). However, in a single-institution setting, it is difficult to achieve sample sizes of 500 or more patients. A prospective, multicenter radiomics study with deep-learning techniques is anticipated to be launched soon.

A limitation of this study is that no prospective radiomics study was found. Moreover, we only include seven studies in this meta-analysis, so evaluation of publication bias was not feasible. Radiomics features may be influenced by image equipment technical settings, reconstruction kernel, contrast infusion speed, tumor delineation ability, and radiomics software (6). A multi-institutional, prospective study should be conducted to further investigate the predictive power of a radiomics study.

\section{Conclusion}

Using radiomics to predict complete pathological response after neoadjuvant chemoradiotherapy in esophageal cancer is feasible.

\section{Conflicts of Interest}

None.

\section{Authors' Contributions}

Concept design: Kao Y.S.; Data collection: Kao Y.S. and Hsu Y.; Statistical analysis: Kao Y.S.; Manuscript writing: Kao Y.S. and Hsu Y. Final approval of manuscript: Kao Y.S. and Hsu Y.

\section{References}

1 Uhlenhopp DJ, Then EO, Sunkara T and Gaduputi V: Epidemiology of esophageal cancer: Update in global trends, etiology and risk factors. Clin J Gastroenterol 13(6): 1010-1021, 2020. PMID: 32965635. DOI: 10.1007/s12328-020-01237-x

2 Semenkovich TR and Meyers BF: Surveillance versus esophagectomy in esophageal cancer patients with a clinical complete response after induction chemoradiation. Ann Transl Med 6(4): 81, 2018. PMID: 29666804. DOI: 10.21037/ atm.2018.01.31

3 Sheikhbahaei S, Trahan TJ, Xiao J, Taghipour M, Mena E, Connolly RM and Subramaniam RM: FDG-PET/CT and MRI 
for Evaluation of Pathologic Response to Neoadjuvant chemotherapy in patients with breast cancer: A meta-analysis of diagnostic accuracy studies. Oncologist 21(8): 931-939, 2016. PMID: 27401897. DOI: 10.1634/theoncologist.2015-0353

4 de Gouw DJJM, Klarenbeek BR, Driessen M, Bouwense SAW, van Workum F, Fütterer JJ, Rovers MM, Ten Broek RPG and Rosman C: Detecting pathological complete response in esophageal cancer after neoadjuvant therapy based on imaging techniques: a diagnostic systematic review and meta-analysis. J Thorac Oncol 14(7): 1156-1171, 2019. PMID: 30999111. DOI: 10.1016/j.jtho.2019.04.004

5 Wesdorp NJ, Hellingman T, Jansma EP, van Waesberghe JTM, Boellaard R, Punt CJA, Huiskens J and Kazemier G: Advanced analytics and artificial intelligence in gastrointestinal cancer: A systematic review of radiomics predicting response to treatment. Eur J Nucl Med Mol Imaging: 2020. PMID: 33326049. DOI: 10.1007/s00259-020-05142-w

6 Park CM: Can artificial intelligence fix the reproducibility problem of radiomics? Radiology 292(2): 374-375, 2019. PMID: 31211633. DOI: 10.1148/radiol.2019191154

7 Moher D, Liberati A, Tetzlaff J, Altman DG and PRISMA group: Preferred reporting items for systematic reviews and metaanalyses: The PRISMA statement. PLoS Med 6(7): e1000097, 2009. PMID: 19621072. DOI: 10.1371/journal.pmed.1000097

8 Zwanenburg A, Vallières M, Abdalah M, Aerts H, Andrearczyk V, Apte A, Ashrafinia S, Bakas S, Beukinga R, Boellaard R, Bogowicz M, Boldrini L, Buvat I, Cook G, Davatzikos C, Depeursinge A, Desseroit M, Dinapoli N, Dinh C, Echegaray S, El naqa I, Fedorov A, Gatta R, Gillies R, Goh V, Götz M, Guckenberger M, Ha S, Hatt M, Isensee F, Lambin P, Leger S, Leijenaar R, Lenkowicz J, Lippert F, Losnegård A, Maier-hein K, Morin O, Müller H, Napel S, Nioche C, Orlhac F, Pati S, Pfaehler E, Rahmim A, Rao A, Scherer J, Siddique M, Sijtsema N, Socarras fernandez J, Spezi E, Steenbakkers R, Tanadini-lang S, Thorwarth D, Troost E, Upadhaya T, Valentini V, Van dijk L, Van griethuysen J, Van velden F, Whybra P, Richter C and Löck S: The image biomarker standardization initiative: Standardized quantitative radiomics for high-throughput image-based phenotyping. radiology 295(2): 328-338, 2020. DOI: 10.1148/radiol.2020191145

9 Kao YS and Hsu Y: Adjuvant contralateral neck irradiation for oral cavity cancer - A systematic review and meta-analysis. Am J Otolaryngol 42(2): 102885, 2021. PMID: 33476968. DOI: 10.1016/j.amjoto.2020.102885

10 Hanley JA and McNeil BJ: The meaning and use of the area under a receiver operating characteristic (ROC) curve. Radiology 143(1): 29-36, 1982. PMID: 7063747. DOI: 10.1148/radiology.143.1.7063747

11 Higgins JP, Thomas J, Chandler J, Cumpston M, Li T, Page MJ and Welch VA: Cochrane handbook for systematic reviews of interventions. John Wiley \& Sons, 2019.

12 Lambin P, Leijenaar RTH, Deist TM, Peerlings J, de Jong EEC, van Timmeren J, Sanduleanu S, Larue RTHM, Even AJG, Jochems A, van Wijk Y, Woodruff H, van Soest J, Lustberg T, Roelofs E, van Elmpt W, Dekker A, Mottaghy FM, Wildberger JE and Walsh S: Radiomics: The bridge between medical imaging and personalized medicine. Nat Rev Clin Oncol 14(12): 749-762, 2017. PMID: 28975929. DOI: 10.1038/nrclinonc. 2017.141

13 Stanzione A, Gambardella M, Cuocolo R, Ponsiglione A, Romeo $\mathrm{V}$ and Imbriaco M: Prostate MRI radiomics: A systematic review and radiomic quality score assessment. Eur J Radiol 129: 109095, 2020. PMID: 32531722. DOI: 10.1016/j.ejrad. 2020.109095

14 Beukinga RJ, Hulshoff JB, van Dijk LV, Muijs CT, Burgerhof JGM, Kats-Ugurlu G, Slart RHJA, Slump CH, Mul VEM and Plukker JTM: Predicting response to neoadjuvant chemoradiotherapy in esophageal cancer with textural features derived from pretreatment ${ }^{18} \mathrm{~F}-\mathrm{FDG}$ PET/CT imaging. J Nucl Med 58(5): 723-729, 2017. PMID: 27738011. DOI: 10.2967/ jnumed.116.180299

15 Beukinga RJ, Hulshoff JB, Mul VEM, Noordzij W, Kats-Ugurlu G, Slart RHJA and Plukker JTM: Prediction of response to neoadjuvant chemotherapy and radiation therapy with baseline and restaging ${ }^{18} \mathrm{~F}$-FDG PET imaging biomarkers in patients with esophageal cancer. Radiology 287(3): 983-992, 2018. PMID: 29533721. DOI: $10.1148 /$ radiol.2018172229

16 Beukinga RJ, Wang D, Karrenbeld A, Dijksterhuis WPM, Faber H, Burgerhof JGM, Mul VEM, Slart RHJA, Coppes RP and Plukker JTM: Addition of HER2 and CD44 to ${ }^{18}$ F-FDG PETbased clinico-radiomic models enhances prediction of neoadjuvant chemoradiotherapy response in esophageal cancer. Eur Radiol, 2020. PMID: 33151397. DOI: 10.1007/s00330-02007439-8

17 Hu Y, Xie C, Yang H, Ho JWK, Wen J, Han L, Chiu KWH, Fu $\mathrm{J}$ and Vardhanabhuti $\mathrm{V}$ : Assessment of intratumoral and peritumoral computed tomography radiomics for predicting pathological complete response to neoadjuvant chemoradiation in patients with esophageal squamous cell carcinoma. JAMA Netw Open 3(9): e2015927, 2020. PMID: 32910196. DOI: 10.1001/jamanetworkopen.2020.15927

$18 \mathrm{Hu}$ Y, Xie C, Yang H, Ho JWK, Wen J, Han L, Lam KO, Wong IYH, Law SYK, Chiu KWH, Vardhanabhuti V and Fu J: Computed tomography-based deep-learning prediction of neoadjuvant chemoradiotherapy treatment response in esophageal squamous cell carcinoma. Radiother Oncol 154: 613, 2021. PMID: 32941954. DOI: 10.1016/j.radonc.2020.09.014

19 van Rossum PS, Fried DV, Zhang L, Hofstetter WL, van Vulpen M, Meijer GJ, Court LE and Lin SH: The incremental value of subjective and quantitative assessment of 18F-FDG PET for the prediction of pathologic complete response to preoperative chemoradiotherapy in esophageal cancer. J Nucl Med 57(5): 691700, 2016. PMID: 26795288. DOI: 10.2967/jnumed.115.163766

20 Hirata A, Hayano K, Ohira G, Imanishi S, Hanaoka T, Murakami $\mathrm{K}$, Aoyagi T, Shuto K and Matsubara H: Volumetric histogram analysis of apparent diffusion coefficient for predicting pathological complete response and survival in esophageal cancer patients treated with chemoradiotherapy. Am J Surg 219(6): 1024-1029, 2020. PMID: 31387687. DOI: 10.1016/ j.amjsurg.2019.07.040

21 Yang Z, He B, Zhuang X, Gao X, Wang D, Li M, Lin Z and Luo $\mathrm{R}$ : CT-based radiomic signatures for prediction of pathologic complete response in esophageal squamous cell carcinoma after neoadjuvant chemoradiotherapy. J Radiat Res 60(4): 538-545, 2019. PMID: 31111948 . DOI: $10.1093 /$ jrr/rrz027

22 Rishi A, Zhang GG, Yuan Z, Sim AJ, Song EY, Moros EG, Tomaszewski MR, Latifi K, Pimiento JM, Fontaine JP, Mehta R, Harrison LB, Hoffe SE and Frakes JM: Pretreatment CT and ${ }^{18}$ F-FDG PET-based radiomic model predicting pathological complete response and loco-regional control following neoadjuvant chemoradiation in oesophageal cancer. J Med 
Imaging Radiat Oncol 65(1): 102-111, 2021. PMID: 33258556. DOI: $10.1111 / 1754-9485.13128$

23 Yip SS, Coroller TP, Sanford NN, Mamon H, Aerts HJ and Berbeco RI: Relationship between the temporal changes in positron-emission-tomography-imaging-based textural features and pathologic response and survival in esophageal cancer patients. Front Oncol 6: 72, 2016. PMID: 27066454. DOI: 10.3389/fonc. 2016.00072

24 Chetan MR and Gleeson FV: Radiomics in predicting treatment response in non-small-cell lung cancer: current status, challenges and future perspectives. Eur Radiol 31(2): 1049-1058, 2021. PMID: 32809167. DOI: 10.1007/s00330-020-07141-9

25 Ursprung S, Beer L, Bruining A, Woitek R, Stewart GD, Gallagher FA and Sala E: Radiomics of computed tomography and magnetic resonance imaging in renal cell carcinoma-a systematic review and meta-analysis. Eur Radiol 30(6): 35583566, 2020. PMID: 32060715. DOI: 10.1007/s00330-02006666-3

26 Rizzo S, Botta F, Raimondi S, Origgi D, Fanciullo C, Morganti AG and Bellomi M: Radiomics: The facts and the challenges of image analysis. Eur Radiol Exp 2(1): 36, 2018. PMID: 30426318. DOI: 10.1186/s41747-018-0068-Z
27 Qazi MA, Vora P, Venugopal C, Sidhu SS, Moffat J, Swanton C and Singh SK: Intratumoral heterogeneity: pathways to treatment resistance and relapse in human glioblastoma. Ann Oncol 28(7): 1448-1456, 2017. PMID: 28407030. DOI: 10.1093/annonc/ $\operatorname{mdx} 169$

$28 \mathrm{Lim} \mathrm{ZF}$ and Ma PC: Emerging insights of tumor heterogeneity and drug resistance mechanisms in lung cancer targeted therapy. J Hematol Oncol 12(1): 134, 2019. PMID: 31815659. DOI: 10.1186/s13045-019-0818-2

29 Cho J, Lee K, Shin E, Choy G and Do S: How much data is needed to train a medical image deep learning system to achieve necessary high accuracy? arXiv:151106348, 2015.

Received February 3, 2021

Revised March 13, 2021

Accepted March 18, 2021 